\title{
EVALUACIÓN DE LEGUMINOSAS HERBÁCEAS FORRAJERAS EN SISTEMAS DE PRODUCCIÓN DE LECHE DE ALTURA
}

\author{
María Mesén Villalobos. ${ }^{1}$, William Sánchez Ledezma. ${ }^{1}$
}

\begin{abstract}
RESUMEN
La presente investigación se realizó en una finca ubicada en el distrito Cot, cantón Oreamuno, provincia Cartago. La topografía de la finca es irregular y se encuentra a una altitud de $2.100 \mathrm{msnm}$. La temperatura y precipitación promedio anual son de $20,7^{\circ} \mathrm{C}$ y $2.121 \mathrm{~mm}$, respectivamente. El objetivo fue evaluar la adaptabilidad de una colección de 15 cultivares de leguminosas de los géneros Vicia, Lotus y Trifolium. El diseño experimental utilizado fue de bloques completos al azar. El análisis estadístico de los resultados se realizó por medio de un análisis de varianza para los forrajes anuales y por medio de una prueba de "t de Student" para los perennes y bianuales conjuntamente, ya que de este grupo sobrevivieron únicamente dos cultivares perennes. Los cultivares anuales presentaron altos rendimientos de materia seca y calidad nutritiva; el análisis de varianza para esos valores demuestra que hay diferencias significativas entre los mismos $(p=0,003)$. El de mayor producción de materia seca fue Vicia villosa Woolly, con 7,1 t/ha/corte y el de menor producción fue el Trifolium alexandrinum Berseen, con 1,5 t/ha/corte. El valor nutritivo fue superior en las leguminosas del género Vicia que en las de Trifolium. Los cultivares perennes y bianuales recibieron cortes cada seis semanas, durante dos años; al último corte los cultivares persistentes fueron, el Lotus corniculatus Makú y el Trifolium repens Arán, ambos perennes, con rendimientos promedio de materia seca de 1,4 y 0,9 t/ha/corte, respectivamente. La prueba de "t de Student" para los valores mencionados demuestra que no hay diferencias significativas entre cultivares $(p=0,32)$. Los valores de proteína cruda oscilaron entre 26,3 y $28,7 \%$. Se concluye que todos los cultivares anuales, se adaptan a la zona en estudio, ya que presentaron altos rendimientos, sin embargo la leguminosa Vicia villosa Woolly mostró el rendimiento más alto. De los cultivares perennes se deben continuar estudiando el Lotus corniculatus Makú. y el Trifolium repens Arán. ya que fueron los únicos que persistieron al cabo de dos años. El testigo local desapareció, no se comportó bien como monocultivo bajo corte, presentó bajos rendimientos e invasión de malezas en los primeros cortes y luego desapareció; sin embargo, en la zona se encuentra en asociación con kikuyo (Pennisetum clandestinum) en forma natural. Los cultivares bianuales no se adaptaron a la zona, presentaron bajos rendimientos, invasión de malezas y poca persistencia. La utilización de forrajes anuales, perennes o ambos en una finca de ganadería de leche, depende de los objetivos y del manejo del sistema de producción en general. Además, se debe seguir investigando con otras leguminosas de uso eventual en la ganadería de leche.
\end{abstract}

Palabras clave: Cultivares, Vicia, Lotus, Trifolium, rendimiento, valor nutritivo.

\section{INTRODUCCIÓN}

La ganadería bovina de leche representa un papel muy importante en la evolución económica y social de Costa Rica, ya que los productos lácteos son básicos en la dieta de la población; además constituyen un rubro de mucha impor- tancia en la economía nacional, logrando llenar la demanda interna y generando divisas provenientes de las exportaciones.

Costa Rica cuenta con la planta de mayor volumen de industrialización de leche en América Central y el Caribe, procesa aproximadamente

1. Instituto Nacional de Innovación y Transferencia en Tecnología Agropecuaria (INTA). Costa Rica. 
$927.000 \mathrm{~kg}$ de leche diarios. Sin embargo, la tendencia mundial de libre comercio requiere de mayores niveles de eficiencia en los sistemas de producción, de manera tal que se pueda competir con precios adecuados y productos de buena calidad en la exportación a otras naciones (Villegas 2004).

En la mayoría de las empresas de ganadería de leche, para la alimentación del ganado se utilizan los forrajes, principalmente gramíneas. Sin embargo, estos no llenan los requerimientos de los animales de mediana y alta producción. En general, los animales que consumen forrajes de baja calidad, sufren deficiencias principalmente de proteína, energía y minerales. Por esta razón, en la alimentación del ganado de leche se utiliza gran cantidad de concentrados, lo que genera un aumento en los costos de producción.

En este sentido, las leguminosas conjuntamente con las gramíneas son los dos grandes órdenes botánicos forrajeros más importantes en el reino vegetal; las leguminosas en monocultivo o en asociación con gramíneas son opciones muy importantes para contribuir a atenuar las deficiencias antes expuestas (Mila 1992). Las leguminosas han sido reconocidas como fuentes de excelente forraje y como mejoradoras de la fertilidad del suelo. Su habilidad para fijar nitrógeno del aire y su alto contenido de proteína y minerales, las hacen indispensables en la alimentación animal (Bernal 1991).

Las especies de leguminosas de los géneros Vicia, Lotus y Trifolium son forrajeras de alto rendimiento y valor nutritivo y la mayoría se adaptan a alturas superiores a los $2.000 \mathrm{msnm}$ (Bernal 1991).

Por lo anteriormente mencionado, se evaluó una colección de leguminosas forrajeras de los géneros mencionados, con el objetivo de buscar variedades de alto rendimiento y valor nutritivo, que se adapten a la zona alta lechera de Costa Rica.

\section{MATERIALES Y MÉTODOS}

El presente trabajo se desarrolló, en una finca ubicada en el distrito Cot, cantón Oreamuno, provincia Cartago, a $83^{\circ} 53^{\prime} 30^{\prime \prime}$ longitud oeste y a $9^{\circ} 57^{\prime}$ latitud norte. La topografía de la finca es irregular y se encuentra a una altitud de $2.100 \mathrm{msnm}$. La temperatura y precipitación promedio anual son de $20,7{ }^{\circ} \mathrm{C}$ y $2.121 \mathrm{~mm}$, respectivamente.

El terreno utilizado había sido cultivado anteriormente con forrajes de piso (Pennisetum clandestinum y Trifolium repens). Geomorfológicamente el suelo es de origen volcánico y taxonómicamente corresponde al orden de los andisoles (Bertsch et al. 1993).

La preparación del suelo fue mecánica, realizando una arada, dos rastreadas y surcando cada $0,5 \mathrm{~m}$.

La siembra se realizó utilizando semilla de una colección de leguminosas forrajeras para clima frío (ver Cuadro 1). Para los forrajes perennes y bianuales se hizo corte de uniformización al inicio de las lluvias del año siguiente y se hicieron evaluaciones de producción de biomasa, altura, cobertura e incidencia de plagas y enfermedades cada seis semanas durante dos años. Los cultivares anuales no se nivelaron ya que se cosechan solamente una vez.

La metodología de evaluación que se utilizó fue una modificación de la utilizada por la Red Internacional de Evaluación de Pastos Tropicales (RIEPT) (Toledo y Schultze-Kraft 1982). Dicha modificación consistió en una disminución en el tamaño de las parcelas y del marco para muestrear el cual fue de 0,5 metros x 1,0 metros, con cuadrículas de 0,25 metros x 0,25 metros. Además, se usó una escala de uno a cuatro para plagas en general, y no se emplearon escalas específicas para insectos y enfermedades como señala la metodología en mención. 
MESÉN Y SÁNCHEZ: Evaluación de leguminosas herbáceas forrajeras en sistemas de producción de leche de altura

Cuadro 1. Cultivares de leguminosas evaluadas. Cartago, Costa Rica. 1996.

\begin{tabular}{|lccc|}
\hline Cultivares & Comportamiento & Simbología & Procedencia ${ }^{1}$ \\
\hline Vicia purpurea Púrpura & Anual & VP & Chile \\
Vicia villosa Woolly & Anual & VV & Australia \\
Lotus corniculatus Dewey & Perenne & LD & E.E.U.U. \\
Lotus corniculatus Makú & Perenne & LM & Australia \\
Trifolium pratense Renegade & Bianual & TR & E.E.U.U. \\
Trifolium alexandrinum Berseen & Anual & TB & E.E.U.U. \\
Trifolium alexandrinum Nico & Anual & TN & Chile \\
Trifolium repens Arán & Perenne & TA & Inglaterra \\
Trifolium repens Italia & Perenne & TI & Chile \\
Trifolium repens Huia & Perenne & TH & Chile \\
Trifolium pratense Safari & Bianual & TS & Australia \\
Trifolium pratense Quiñequeli & Bianual & TQ & Chile \\
Trifolium subterraneum Clare & Perenne & TC & Chile \\
Trifolium subterraneum Mount Barquer & Perenne & TM & Chile \\
Trifolium repens (Testigo con material & & & Costa Rica \\
vegetativo) & Perenne & TL & \\
\hline
\end{tabular}

${ }^{1}$ Bradley, R. 1996. Semillas forrajeras (entrevista). FUDESEMILLAS. San José, CR. Comunicación personal.

\section{Descripción de la unidad experimental}

La parcela experimental midió $6 \mathrm{~m}^{2}$ con tres hileras de 3,0 $\mathrm{m}$ de largo y distanciadas entre ellas a 0,5 m. Los muestreos se tomaron en la hilera central, dejando los otros surcos como efecto de borde.

\section{Diseño experimental}

El diseño experimental utilizado fue de bloques completos al azar con tres repeticiones.

\section{Análisis de los resultados}

Los resultados obtenidos en cuanto a materia seca se analizaron por medio de un análisis de varianza para los forrajes anuales y por medio de una prueba de "t de Student" para los perennes y bianuales, ya que de estos últimos sobrevivieron dos cultivares perennes. Se utilizó el software de estadística y biometría Info Stat 2001 (UNC 2001).

\section{Fertilización}

Las dosis aplicadas fueron: $50,50,20$ y $20 \mathrm{~kg} /$ ha de $\mathrm{P}_{2} \mathrm{O}_{5}, \mathrm{~K}_{2} \mathrm{O}, \mathrm{Mg}$ y S. El fósforo, magnesio y azufre se incorporaron al suelo al momento de la siembra, el potasio se aplicó fraccionado, con $1 / 3$ de lo recomendado cuatro semanas después de la siembra, 1/3 después del corte de uniformización y el último tercio al cabo de dos cortes (Toledo y Schultze-Kraft 1982).

\section{Variables evaluadas}

\section{Producción de biomasa y calidad nutritiva}

Como se mencionó anteriormente, las evaluaciones para los forrajes perennes y bianuales se realizaron cada seis semanas después del corte de nivelación; la altura de corte fue de aproximadamente $5,0 \mathrm{~cm}$ sobre el nivel del suelo. Los cortes se efectuaron a razón de un metro lineal en la hilera central de cada parce- 
la, dejando sin cortar los extremos, como efecto de borde (Roig 1989). Los forrajes anuales se cosecharon en forma similar, pero se utilizó el metro cuadrado para los forrajes del género Vicia.

El material de la hilera central se pesó en verde y luego una sub-muestra de $500 \mathrm{~g}$ se envió al laboratorio para la determinación del contenido de materia seca (MS), proteína cruda (PC) y digestibilidad in vitro de la materia seca (DIVMS).

\section{Cobertura}

Esta variable se evaluó como porcentaje del área que no presentó suelo desnudo. Las evaluaciones se efectuaron cada seis semanas coincidiendo con los muestreos de rendimiento. Para este propósito, se utilizó la metodología propuesta por la RIEPT (Toledo y Schultze-Kraft 1982) con las modificaciones antes expuestas.

\section{Altura}

La variable altura se midió con la misma frecuencia que la cobertura.

Para esta medición, se tomó la altura desde el nivel del suelo hasta el punto más alto de la planta, sin estirarla y sin considerar la inflorescencia (Toledo y Schultze-Kraft 1982).

\section{Plagas (Insectos y enfermedades)}

Se utilizó una escala de 1 a 4, en la cual:

$1=$ Presencia ( $5 \%$ del follaje afectado)

$2=$ Daño leve $(5 \%-20 \%$ afectado $)$

$3=$ Daño moderado (20\% - 40\% afectado)
4 = Daño grave (más de 40\% afectado) (Adaptado de Calderón 1982 y Lenne 1982).

\section{RESULTADOS Y DISCUSIÓN}

\section{Análisis de suelo}

El análisis de suelo antes de la siembra (Cuadro 2) presenta valores adecuados de $\mathrm{pH}$ y aluminio, como consecuencia el porcentaje de acidez, 8,8 es adecuado. Los contenidos de $\mathrm{Ca}, \mathrm{Mg}, \mathrm{K}, \mathrm{P}$, los microelementos y las relaciones $(\mathrm{Ca} / \mathrm{Mg}, \mathrm{Mg} / \mathrm{K}, \mathrm{Ca}+\mathrm{Mg} / \mathrm{K}$ y $\mathrm{Ca} / \mathrm{K})$ se encuentran en un rango óptimo, mientras que el $\mathrm{Fe}$ se encuentra en un nivel alto (Bertsch 1987). En relación con la textura, se puede concluir que por ser un suelo franco presenta buena infiltración, mediana capacidad de retención de humedad y buena aireación. En cuanto al contenido de materia orgánica, el suelo es apto para el desarrollo de los forrajes ya que es un valor característico de un suelo agrícola (Núñez 1985).

\section{Cultivares anuales}

Los cultivares anuales fueron cosechados al estar en floración plena (aproximadamente 176 días de crecimiento y durante la época lluviosa), su comportamiento fue bueno, los altos rendimientos de materia seca y la ausencia de plagas demuestran la adaptabilidad de los mismos a la zona. La leguminosa Vicia villosa Woolly, fue el cultivar de mayor producción de biomasa en base seca, con 7,1 t/ha valor similar al obtenido por Mesén y Sánchez 2005, y superior a 4,3 t/ha reportado por Rodríguez (1979), para Vicia villosa Namoy. El Trifolium alexandrinum Berseen fue el de menor producción con 1,5 t/ha.

Cuadro 2. Resultado del análisis de suelo antes de la siembra. Cartago, Costa Rica. $1996 .{ }^{1}$

\begin{tabular}{ccccccccccccc}
\hline & \multicolumn{4}{c}{ Meq/100ml suelo } & \multicolumn{4}{c}{ Ug/ml suelo } & Textura & $\begin{array}{c}\text { Materia } \\
\text { Orgánica }\end{array}$ \\
\hline $\mathrm{pH}$ & $\mathrm{Al}$ & $\mathrm{Ca}$ & $\mathrm{Mg}$ & $\mathrm{K}$ & $\mathrm{P}$ & $\mathrm{Zn}$ & $\mathrm{Mn}$ & $\mathrm{Cu}$ & $\mathrm{Fe}$ & & \\
6,0 & 0,15 & 7,8 & 2,0 & 1,5 & 15,0 & 19,9 & 13,0 & 32,0 & 208 & Franco & 11,3 \\
\hline
\end{tabular}

${ }^{1}$ Análisis de suelo realizado por el Laboratorio de Suelos, Foliares y Aguas del INTA. 
MESÉN Y SÁNCHEZ: Evaluación de leguminosas herbáceas forrajeras en sistemas de producción de leche de altura

Los valores de cobertura y altura oscilaron entre 75 y $100 \%$ y entre 42 y $60 \mathrm{~cm}$, respectivamente. La calidad nutritiva fue superior en los cultivares del género Vicia (Cuadro 3).
El análisis de varianza para los valores de materia seca demuestra que hay diferencias sig-

nificativas entre los mismos $(p=0,003)$.

Cuadro 3. Comportamiento de las leguminosas anuales al estar en floración. Cartago, Costa Rica. 1998.

\begin{tabular}{cccccc}
\hline Cultivar & $\begin{array}{c}\text { Cobertura } \\
(\%)\end{array}$ & $\begin{array}{c}\text { Altura } \\
(\mathbf{c m})\end{array}$ & $\begin{array}{c}\text { PC, } \\
(\%)\end{array}$ & DIVMS, (\%) & $\begin{array}{c}\text { MS, } \\
\text { t/ha/corte }\end{array}$ \\
\hline VP & 100 & 60 & 22,0 & 63,2 & 4,6 \\
VV & 100 & 57 & 24,0 & 65,2 & 7,1 \\
TB & 90 & 39 & 16,2 & 66,0 & 1,5 \\
TN & 75 & 42 & 13,7 & 63,3 & 4,3 \\
\hline
\end{tabular}

\section{Cultivares perennes y bianuales}

De los once cultivares evaluados sobrevivieron únicamente dos cultivares perennes al cabo de dos años: el Lotus corniculatus Makú, y el Trifolium repens Arán con rendimientos promedio de materia seca de 1,4 y 0,9 t/ha/corte, respectivamente (Cuadro 4). Los demás cultivares entre los cuales habían perennes y bianuales (Cuadro 1) presentaron bajos rendimientos e invasión de malezas y al final de la prueba desaparecieron, no se adaptaron a la zona en estudio.

La prueba de "t de Student" para los dos cultivares perennes persistentes al cabo de dos años, demuestra que no hay diferencias significativas entre los mismos $(p=0,32)$. Los valores de proteína cruda oscilaron entre 26,3 y $28,7 \%$ (Cuadro 4). Los valores promedio de altura fueron 30 y $23 \mathrm{~cm}$, los de cobertura 80 y $65 \%$ para Lotus corniculatos Makú, y el Trifolium repens Arán respectivamente. No hubo presencia de insectos ni enfermedades importantes.

El testigo local desapareció, no se comportó bien como monocultivo bajo corte, presentó bajos rendimientos e invasión de malezas en los primeros cortes y luego desapareció; sin embargo, en la zona se encuentra en asociación con kikuyo (Pennisetum clandestinum) en forma natural.

Cabe recalcar que los forrajes perennes a diferencia de los anuales (solamente un corte), se cosechan varias veces al año y se mantienen en producción durante varios años sin necesidad de sembrar en cada período. En los forrajes perennes de este estudio se realizaron ocho cortes por año, los cuales proporcionaron aproximadamente 11,2 y 7,2 toneladas de materia seca por hectárea por año para Lotus corniculatus Makú, y Trifolium repens Arán respectivamente; valores superiores a los obtenidos con los forrajes anuales, calidad nutritiva similar y costo inferior.

No es conveniente precisar en términos generales, cuál de los dos tipos de forraje es el recomendable, ya que el uso de uno u otro o de ambos, depende del sistema de producción y de sus objetivos. Los forrajes anuales podrían ser una buena opción en fincas de ganadería de leche que producen otros cultivos y ser utilizados en rotación con los otros cultivos, para manejarlos como forraje de corte fresco o conservado, durante períodos críticos cuando hay escasez de forrajes de piso.

En el caso de los forrajes anuales cuando la siembra se hace después de cosechar otro cultivo, se evita el uso de herbicidas, se aprovechan los residuos de fertilizante y se reduce el uso de mano de obra. Además las leguminosas 
incorporan nitrógeno al suelo lo cual beneficiará el próximo cultivo.

Cuadro 4. Comportamiento de las leguminosas perennes o bianuales durante el período de corte. Cartago, Costa Rica.1998.

\begin{tabular}{lccccc}
\hline \multicolumn{2}{c}{ Época Lluviosa } & \multicolumn{2}{c}{ Época seca } & \\
Cultivar & Proteína, (\%) & $\begin{array}{c}\text { Materia seca } \\
\text { (t/ha/corte) }\end{array}$ & Proteína (\%) & $\begin{array}{c}\text { Materia seca } \\
\text { (t/ha/corte) }\end{array}$ & $\begin{array}{c}\text { Promedio } \\
\text { anual (t/ha/ } \\
\text { corte) }\end{array}$ \\
\hline LD & $\mathrm{P}$ & & & & \\
LM & 28,6 & 1,8 & 28,7 & 0,9 & 1,4 \\
TR & 24,6 & 1,6 & $\mathrm{P}$ & & \\
TA & 28,0 & 1,1 & 26,3 & & \\
TI & $\mathrm{P}$ & & & & \\
TH & $\mathrm{P}$ & & & & \\
TS & $\mathrm{P}$ & & $\mathrm{P}$ & \\
TQ & 25,8 & 0,9 & & \\
TC & $\mathrm{P}$ & & & \\
TM & $\mathrm{P}$ & $\mathrm{P}$ & & & \\
Testigo & & & & & \\
\hline
\end{tabular}

$\mathrm{P}=$ Perdido.

\section{CONCLUSIONES Y RECOMENDACIONES}

Teniendo en consideración las condiciones bajo las cuales se llevó a cabo el ensayo, se pueden formular las siguientes conclusiones y recomendaciones:

1. Los cuatro cultivares anuales dieron buenos resultados, las altas tasas de crecimiento por hectárea y la ausencia de plagas, demuestran la adaptabilidad de los mismos a la zona.

2. De los cultivares anuales el de mayor producción fue Vicia villosa Woolly, y el de menor producción fue Trifolium alexandrinum Berseen.

3. Los cultivares bianuales presentaron bajos rendimientos e invasión de malezas, lo cual demuestra que no se adaptan a la zona en estudio.

4. Los cultivares perennes (Lotus corniculatus Makú y el Trifolium repens Arán) no presen- taron problemas de plagas, lograron altas tasas de crecimiento, adecuado valor nutritivo y buena persistencia lo que demuestra su adaptabilidad a la zona. Los demás cultivares perennes evaluados no se adaptan como monocultivo bajo corte.

5. La utilización de forrajes anuales, perennes o ambos en una finca de ganadería de leche, depende de los objetivos y del sistema de producción en general.

6. Se debe continuar investigando con los cultivares sobresalientes en asociación con gramíneas.

7. Se debe continuar investigando con otras leguminosas de uso potencial en sistemas de producción de leche de altura.

\section{AGRADECIMIENTO}

Los autores expresan su agradecimiento al Doctor Danilo Pezo Q., y a los Ingenieros Luis Villegas Z. y Beatriz Molina B. por la orientación científica que le brindaron a este trabajo. 


\section{LITERATURA CITADA}

Bernal, J. 1991. Pastos y forrajes tropicales. 2 Ed. Colombia. Editorial Banco ganadero. $543 \mathrm{p}$.

Bertsch, F.; Mata, R.; Henríquez, C. 1993. Características de los principales órdenes de suelos presentes en Costa Rica. Congreso Nacional Agropecuario y de Recursos Naturales. U.C.R. Costa Rica. 78 p.

1987. Manual para interpretar la fertilidad de los suelos de Costa Rica. 2 Ed. Costa Rica. Universidad de Costa Rica. 82 p.

Calderón, M. 1982. Evaluación del daño causado por insectos. In: Toledo, J.M. Manual para la evaluación agronómica. Red Internacional de Evaluación de Pastos Tropicales. (RIEPT), CIAT, Cali, Colombia. p. 45-56.

UNC (Universidad de Córdoba, AR). 2001. Info Stat: Software de Estadística y Biometría. Info Stat 2001. Versión 1.0 .

Lenne, J. 1982. Evaluación de enfermedades en pastos tropicales. In: Toledo, J.M. Manual para la evaluación agronómica. Red Internacional de Evaluación de Pastos Tropicales. (RIEPT), CIAT, Cali, Colombia. p. 57-72.

Mila, A. 1992. Las especies forrajeras en el reino vegetal. In: Pastos y forrajes para Colombia. Editorial Banco Ganadero. $3^{a}$ Edición. Colombia. p.7.
Mesén, M.; Sánchez W. 2005. Evaluación de leguminosas herbáceas de uso potencial en sistenas de producción de leche de altura. Alcances Tecnológicos. no.1:79-84.

Núñez, J. 1985. Fundamentos de Edafología. 2 Ed. Costa Rica. UNED. 185 p.

Roig, C. A. 1989. Evaluación preliminar de 200 accesiones de leguminosas forrajeras tropicales en el ecosistema de Bosque Tropical Lluvioso en Costa Rica Guápiles. Tesis MSc.Turrialba C.R, CATIE. 179 p.

Rodriguez, H. 1979. Determinación de la densidad de siembra de la Veza vellosa como abono verde y su efecto en la producción de sorgo de grano. Tesis Ing. Agr. México. Universidad Autónoma de Nuevo León. Facultad de Agronomía. 31 p.

Toledo, J. M.; Schultze - Kraft, R. 1982. Metodología para la evaluación agronómica de pastos tropicales. In: Toledo, J.M. Manual para la Evaluación Agronómica. Red Internacional de Evaluación de Pastos Tropicales (RIEPT) CIAT, Calí, Colombia. p. 91-109.

Villegas, L. 2004. Factores determinantes de la productividad de fincas lecheras en pastoreo. In: Memoria del seminario de ganadería de leche. Departamento de Transferencia. INTA. Costa Rica. 
ALCANCES TECNOLÓGICOS, AÑO 5, NÚMERO 1 